\title{
Dokumentationsversäumnisse Kündigung des Arztes berechtigt
}

Nach Auffassung des Sächsischen Landesarbeitsgerichts (Urteil vom 22.09.2010 Az.: 2 Sa 56/10) war die Kündigung eines leitenden Arztes aufgrund von unterlassener DRG-Kodierung und Dokumentationsversäumnissen rechtswirksam. Der Kündigung waren 2 einschlägige Abmahnungen vorausgegangen.

\section{Der Fall}

Bei dem betroffenen Arzt handelt es sich um einen Facharzt für Chirurgie und Unfallchirurgie, der seit Januar 2003 als leitender Arzt in einer Klinik tätig war. Im Dienstvertrag des Arztes befand sich folgende Regelung: „Der Arzt ist insbesondere für eine richtige und vollständige Kodierung und Dokumentation der für die Eingruppierung in einem deutschen DRG-System erforderlichen Diagnosen und Prozeduren nach Maßgabe der jeweils gültigen Deutschen Kodierrichtlinien verantwortlich. Er hat der Krankenhausverwaltung alle erforderlichen Unterlagen zur Verfügung zu stellen.“

\section{Zwei Abmahnungen wegen fehlender Dokumentation}

Mit Schreiben vom 16.04.2009 mahnte die Krankenhausträgerin den Arzt ab, weil er eine am 06.11.2008 durchgeführte Operation einer Patientin bis zum 10.12.2008 nicht dokumentiert hatte. Die Eingabe der notwendigen Daten sei erst anhand der Patientenakte von einem Mitarbeiter des Medizincontrollings zur Sicherung der Erlöse und erforderlichen Rechnungslegung an den Kostenträger in das System eingepflegt worden. Darüber hinaus habe der Arzt seinen Dienstvorgesetzten belogen, weil er im Rahmen einer Besprechung auf Nachfrage des Chefarztes nach diesem Fall erklärt habe, die Prozeduren selbst eingepflegt zu haben. Dem Arzt wurde Gelegenheit zur schriftlichen Stellungnahme bis zum 27.04.2009 gegeben.

Mit Schreiben vom 24.04.2009 wurde der Arzt zum zweiten Mal abgemahnt. Nun wurde ihm vorgeworfen, die Operationen eines Patienten am 28.10.2008 und am 30.11.2008 nicht ausreichend dokumentiert zu haben. Die vom MDK abverlangte Stellungnahme erweise sich als wider- sprüchlich, insbesondere zum OP-Bericht und entspreche nicht den qualitativen Normen einer kompetenten fachlichen Bewertung. Das MDK-Gutachten vom 31.03.2009 sei zu dem Ergebnis gekommen, dass beide Operationen $\mathrm{zu}$ einem Fall hätten zusammengeführt werden müssen. Dadurch seien der Krankenhausträgerin Erlöseinbußen entstanden. Der Arzt erhielt Gelegenheit zur Stellungnahme zu dieser Abmahnung bis 11.05.2009. Auf beide Abmahnungen reagierte der Kläger jeweils mit Schreiben vom 11.05.2009. Hinsichtlich der ersten Abmahnung bat der Arzt um das vollständige Protokoll seines Computers sowie des Programms zur Überprüfung der Arbeitsvorgänge und Arbeitsschritte, um zu den Vorwürfen Stellung nehmen zu können. Bezüglich der zweiten Abmahnung vertrat der Arzt die Auffassung, beide OPBerichte seien eindeutig und klar.

\section{Kündigung wegen unterlassenem DRG-Eintrag}

Am 27.04.2009 operierte der Kläger einen Patienten an der Wirbelsäule. Der Patient wurde am 05.05.2009 entlassen. Die Notwendigkeit der Operation des Patienten ergab sich aus der Einweisungsdiagnose, die bei der Aufnahme des Patienten in die Krankenakte erstellt und für das Medizincontrolling bei der Prüfung der Abrechnungsunterlagen ersichtlich war. Der Arzt hatte bezüglich dieses Patienten in den Abrechnungsunterlagen in der vorgesehenen Spalte „OP“ Prozeduren über eine Operation einzutragen, was er aber unterlassen hatte. Dies fiel dem Mitarbeiter des Medizincontrollings bei der Durchsicht der Abrechnungsunterlagen am 05.05.2009 auf. In Abstimmung mit dem Chefarzt wurde die fehlende Kodierung durch den Mitarbeiter des Medizincontrollings vorgenommen, sodass das Krankenhaus in den Stand versetzt wurde, einen Betrag in Höhe von 3428,00 Euro abzurechnen. Nach Anhörung des für das Krankenhaus errichteten Betriebsrats erklärte die Krankenhausträgerin dem Arzt gegenüber die streitgegenständliche Kündigung. Hiergegen und gegen die beiden Abmahnungen klagte der Arzt. Er bezweckte damit, dass festgestellt wird, dass sein Arbeitsverhältnis nicht aufgelöst wurde. Zudem wollte er erreichen, dass die Abmahnungen für gegenstandslos erklärt und aus seiner Personalakte entfernt werden. Dies alles verfolgt er mit dem Ziel, zu unveränderten Bedingungen als leitender Arzt weiterbeschäftigt zu werden. Das erstinstanzliche Arbeitsgericht gab zunächst dem Arzt Recht. Gegen das Urteil ging die Krankenhausträgerin in Berufung zum Sächsischen Landesarbeitsgericht. Die Krankenhausträgerin hält sowohl am Kündigungsgrund als auch an den den Abmahnungen zugrunde liegenden Sachverhalten fest. Ihrer Auffassung nach hätte das Arbeitsgericht die Klage abweisen müssen.

\section{Landesarbeitsgericht hebt obsie- gendes Urteil für den Arzt auf}

Mit Urteil vom 22.09.2010 gab das Sächsische Landesarbeitsgericht nunmehr der Krankenhausträgerin Recht. Das Arbeitsverhältnis ist aufgelöst worden, da die Kündigung rechtswirksam war. Darüber hinaus muss die Krankenhausträgerin auch nicht die angegriffenen Abmahnungen für gegenstandslos erklären und aus der Personalakte entfernen, denn auch die Abmahnungen sind nach Auffassung des Landesarbeitsgerichts wirksam.

\section{Arzt verletzte seine Vertragspflich- ten - Kündigung wirksam}

Nach Auffassung des Landesarbeitsgerichts ist die Kündigung gerechtfertigt, weil sie durch Gründe, die im Verhalten des Klägers liegen, bedingt ist. Derartige Gründe können insbesondere dann vorliegen, wenn ein Arbeitnehmer seinen Arbeitsvertrag dadurch verletzt, dass er seinen sich aus diesem Vertrag ergebenden Pflichten nicht oder nicht ausreichend nachkommt. Üblicherweise wird ihm dies durch Wahrung der Verhältnismäßigkeit der Vertragsbeendigung vorab durch den früheren fruchtlosen Ausspruch einer oder mehrerer Abmahnungen vor Augen geführt worden sein müssen, welche die Vertragsbeendigung wegen eines dem Kündigungssachverhalt vergleichbaren Sachverhalts für den Wiederholungsfall in Aussicht gestellt haben. Nach Auffassung der Richter hat der Kläger seine arbeitsvertraglichen Pflichten verletzt, wurde hierfür wirksam abgemahnt und die abgemahnte Arbeitspflichtverletzung wurde durch einen gleichzusetzenden Arbeitsvertragsverstoß wiederholt. Ab dem Zeitpunkt der 
Operation des Patienten am 27.04.2009 sei der Kläger seiner arbeitsvertraglichen Rechtspflicht zur Dokumentation nicht nachgekommen. Für diesen am 05.05.2009 zu entlassenden Patienten war bis zum Zeitpunkt seiner Entlassung an diesem Tag nicht aufgefallen, dass seitens des Arztes in der hierfür in den Abrechnungsunterlagen vorgesehenen Spalte „OP“ keinerlei Prozeduren über die Operation eingetragen worden waren. Unerheblich sei, dass und aus welchen Gründen der Arzt die fehlende DRG-Kodierung nicht erkennen konnte. Maßgeblich war nur, dass sie fehlte. Daran ändere sich auch nichts dadurch, dass die Krankenhausträgerin aus wirtschaftlichen Gründen ein Medizincontrolling eingerichtet habe und auch im inkriminierten Fall der Medizincontroller tätig und fündig geworden sei. Denn seine Aufgabe bestehe ausweislich des vom Arzt selbst vorgelegten Auszugs aus der Internetseite der Krankenhausträgerin nicht darin, über arbeitsvertragliche Sanktionen einer unterlassenen Kodierung nachzudenken, solche Sanktionen anzuregen oder gar eine Entscheidung über sie herbeizuführen. Vielmehr habe er Feststellungen zu treffen, aus denen Dritte Schlüsse für die Abrechnung gegenüber den Kostenträgern und/oder hinsichtlich der arbeitsvertraglich verantwortlichen Person ziehen können. Die Krankenhausträgerin sei auch nicht gehalten gewesen, den Arzt nach dem Aufdecken des - neuerlichen und abgemahnten Vertragsverstößen gleichartigen Arbeitsvertragsverstoßes erneut an seine arbeitsvertraglichen Pflichten zu erinnern, ihm zunächst eine Nachfrist zur Dokumentation zu setzen und/oder erneut eine Abmahnung zu erklären. Vielmehr sei der Kläger bereits zweimal wirksam und eindringlich abgemahnt worden.

Die Abmahnung vom 16.04.2009 sei gerechtfertigt. Jedenfalls treffe es zu, dass der Arzt vom Leiter des Medizincontrollings am 05.12.2008 per E-Mail gemahnt wurde, die Prozeduren für den geschilderten Fall der Patientin nicht eingetragen zu haben, weshalb die Abrechnung für den Monat November 2008 nicht abgeschlossen werden könne. Dieser Aufforderung sei der Kläger nicht nachgekommen. Unstreitig sei auch, dass die Eingabe der notwendigen Daten anhand der Patientenakte von einem Mitarbeiter des Medizincontrollings als Kodierfachkraft zur Sicherung der Erlöse und der erforderlichen Rechnungslegung gegenüber dem Kostenträger in das System eingepflegt worden seien und von diesem hierbei die Eingabe eindeutig gekennzeichnet gewesen sei. Zum Zeitpunkt der Anmahnung durch das Medizincontrolling - und nicht erst in Reaktion auf die erteilte Abmahnung - hätte es der Arzt in der Hand gehabt, den Vorgang aufzuklären und insbesondere das vollständige Protokoll seines Computers und des Programms zur Überprüfung der Arbeitsvorgänge und Arbeitsschritte anzufordern. Korrekt war nach Auffassung der Richter auch die zweite Abmahnung mit Schreiben vom 24.04.2009. Vorgehalten werde dem Arzt das Fehlen von Hinweisen zur Anamnese und genauen Indikation hinsichtlich des am 28.10.2008 operierten Patienten, welchen Vorwurf der Arzt so nicht in Abrede gestellt habe. In Abrede habe der Kläger insbesondere auch nicht gestellt, dass im OP-Bericht die zweite Operation des Patienten am 30.11.2008 betreffend keine als Voraussetzung für eine komplikationslose Abrechnung notwendige Bezugnahme zur vorausgehenden Operation hergestellt wurde. Immerhin sei nach Mitteilung der kostentragenden Krankenkasse für den Gutachter im Hause des MDK die Wiederaufnahme des Patienten am 13.11.2008 als Komplikation zum Aufenthalt für den Zeitraum vom 27.10.2008 bis 11.11.2008 zu werten. Ob medizinisch zutreffend oder nicht, habe jedenfalls das Verhalten des Arztes dazu geführt, dass 2 Operationen mit den daraus sich ergebenden Erlöseinbußen zu einem Fall haben zusammengeführt werden müssen.

\section{Interessensabwägung zu Lasten des Arztes}

Die abschließend vorzunehmende Interessensabwägung zwischen dem Bestandsschutz des Arztes gegenüber dem Interesse des Krankenhausträgers, sich von dem Mitarbeiter zu lösen, ging zu Lasten des Arztes aus. Die Richter vertraten dabei die Auffassung, dass der Arzt die wirtschaftlichen Interessen der Krankenhausträgerin am Betrieb eines Krankenhauses ausblende, indem er insinuiere, es handele sich um Einzelfälle mit geringem Schadenspotenzial, wobei der Schadenseintritt durch das dafür vorhandene Medizincontrolling auch noch habe verhindert werden können. Damit stelle der Kläger eine (seine) zentrale Verpflichtung aus dem die Parteien verbindenden Arbeitsvertrag jedenfalls hinsichtlich des
Kündigungssachverhalts sowie der Abmahnungssachverhalte schlicht in Frage. Gemessen daran sei die Krankenhausträgerin nicht gehalten, es auf Wiederholungen oder gar unentdeckte Vertragsverstöße ankommen zu lassen. Insbesondere müsse sich die Krankenhausträgerin nicht darauf verweisen lassen, dass das Medizincontrolling die Funktion habe, ihn an die Erfüllung seines Arbeitsvertrages zu erinnern. Auch wenn dies die Aufgabe des Medizincontrollings sein sollte oder es sich um eine wünschenswerte Aufgabe dieses Controllings handele, vermöge keine Kontrolle die in Person des Arbeitnehmers zu leistende Dienstpflicht zu ersetzen. Das Gericht geht sogar noch weiter und stellt fest, dass selbst wenn man die hier in Rede stehende arbeitsvertragliche Verpflichtung des Klägers als bloße nicht einklagbare Obliegenheit einstufen würde, sich daran nichts ändern würde, dass die negativen Konsequenzen der Obliegenheitsverpflichtung der Arzt zu tragen hätte. Insbesondere dürfe er nicht auf eine Kontrolle verweisen, die ebenso wie er - vertragswidrig versagen könnte. Die allein durch das Controlling letztlich verhinderten Schäden sind auch nicht von untergeordneter Bedeutung. In der Größenordnung ergeben alleine die Kündigungssachverhalte sowie die beiden abgemahnten Sachverhalte einen Wert von ca. 5 monatlichen Grundvergütungen des Klägers oder den des Jahreslohns anderer Beschäftigter, welche Beträge nur durch ordentliche Dokumentation und Abrechnung erwirtschaftet werden können. Nachdem die Krankenhausträgerin gegen die Arbeitsleistungen des Arztes keine fachlichmedizinischen Einwendungen erhob, könne der Kläger ungeachtet seines Lebensalters und seiner Unterhaltsverpflichtungen sowie auch unter Berücksichtigung des langjährigen Arbeitsverhältnisses zwischen den Parteien mit Blick auf den Arbeitsmarkt für qualifizierte Mediziner auf eine anderweitige Beschäftigung am Arbeitsmarkt verwiesen werden. Die Revision wurde nicht zugelassen.

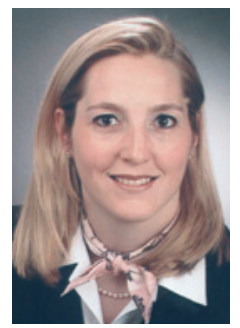

Korrespondenz Dr. iur. Isabel Häser Rechtsanwältin Ehlers, Ehlers und Partner Widenmayerstr. 29 80538 München 